\title{
The Challenge of Artificial Intelligence to China's Penal System
}

\author{
Di Ye \\ Institute of Criminal Justice \\ Zhongnan University of Economics and Law \\ Wuhan, China
}

\begin{abstract}
In the field of criminal law, the recognition of artificial intelligence technology and criminal subjects is considered as one of the key issues. Considering about China's penal system, AI cannot be regulated effectively. Therefore, we must not only consider the effective means of punishing artificial intelligence in the future, but also take a deep consideration into how to effectively regulate artificial intelligence.
\end{abstract}

Keywords-artificial intelligence; criminal law; China's penalty system

\section{INTRODUCTION}

Since the rapid development of information technology in the 21st century, Artificial intelligence technology has revived people's hot discussion. Whether it is through deep learning technology to defeat all the top human players in the Go which is considered to be the most representative of human intelligence, or to determine the personal content of each person through big data analysis to determine the push of the new website, all indicate that artificial intelligence technology has "flied into the houses of ordinary people". But the rapid development of science and technology has also brought about people's worries. This worry is rooted in the thinking of human destiny, once artificial intelligence really exists and even surpasses human intelligence, what the consequence might be? In particular, the legal researchers' reflection of the possible risks of artificial intelligence is the most representative.

Based on the nodes of the development of the artificial intelligence technology, in the field of artificial intelligence, especially the legal qualification of the intelligent artificial life, the copyright of the artificial intelligence works, and the privacy protection, etc, has caused extensive discussion. In the field of criminal law, such as artificial intelligence and sentencing, artificial intelligence and criminal doctrine of liability fixation, artificial intelligence and medical accident crime are of great significance.

\section{THE BACKGROUND OF THE PROBLEM}

\section{A. The Introduction to Artificial Intelligence}

The so-called artificial intelligence, that is, Artificial Intelligence, abbreviated as Artificial intelligence. It is the study, Development Used for simulation, extend and extended intelligent. A new technical science of theory, methods, techniques and application systems.

Thought-capable artificial beings appeared as storytelling devices in antiquity, and have been common in fiction, as in Mary Shelley's Frankenstein or Karel Čapek's R.U.R (Rossum's Universal Robots). These characters and their fates raised many of the same issues now discussed in the ethics of artificial intelligence.

The study of mechanical or "formal" reasoning began with philosophers and mathematicians in antiquity. The study of mathematical logic led directly to Alan Turing's theory of computation, which suggested that a machine, by shuffling symbols as simple as "0" and "1", could simulate any conceivable act of mathematical deduction. This insight, that digital computers can simulate any process of formal reasoning, is known as the ChurchTuring thesis. Along with concurrent discoveries in neurobiology, information theory and cybernetics, this led researchers to consider the possibility of building an electronic brain. Turing proposed that "if a human could not distinguish between responses from a machine and a human, the machine could be considered "intelligent". The first work that is now generally recognized as Artificial intelligence was McCullouch and Pitts' 1943 formal design for Turingcomplete "artificial neurons".

Objectively, the discussion on how to regular Artificial intelligence through crime law is outstrip, due to considering the Artificial intelligence development situation, at present stage, it is hard to say that artificial intelligence technology does not really give its carrier complete intelligence. Therefore, under the vision of the criminal law, the principle of liability on product damage liability can be used to solve the problem that Artificial Intelligence may bring. However, if we start from the perspective of penalty system, the significance of thinking ahead is enough to highlight. Artificial Intelligence technology is developing continuously. Artificial Intelligence can be punished in the future. It should be punished as a more reasonable forecast Therefore, the question of how to punish is more needed to be thought under the premise of reasonable prediction. 


\section{B. The Challenge of Artificial Intelligence to the Determination of the Legal Subject}

The core problem of artificial intelligence research is "intelligence", and intelligence is also the most important feature that people have. What kind of artificial intelligence can be recognized as a "person", and there is a different understanding of artificial intelligence researchers on this issue, and as a result, many schools have emerged. At the same time, this issue is also a reflection on our human beings. What kind of intelligent entities can be called "people". Artificial Intelligence can be studied from different perspective. On the one hand, the result of the development of science and technology, people can gradually reveal the mystery of nature; on the other hand, it is also the process of understanding people themselves. We realize human complex computing, graphic recognition, emotional likes and dislikes, rational reasoning, target selection, etc. on computer systems to understand human beings in the way of computer science and engineering. Therefore, artificial intelligence can become a discipline that crosses many disciplines and is one of the most closely related disciplines of humanities and social sciences, such as philosophy, logic, and computer science, as well as cognitive science, brain science, and so on. The core issue of this crossover is centered around the "human" related proposition. The impact of artificial intelligence on the law also stems from the fact that artificial intelligence can be recognized as a "human" problem. In a certain sense, the research of artificial intelligence is to create a new social activity subject. These activities can have the intellectual knowledge and ability of a part of human beings, and may also possess all the intellectual and abilities of human beings. Nowadays, artificial intelligence is becoming more and more oriented towards the development of all human intelligence, that is, whether general artificial intelligence can be recognized as a "person." Many of the things created by artificial intelligence research and development, we also need to determine the system norms, and the law is different for their qualitative, the corresponding applicable legal rules are different.

The pre-link of the law, and for artificial intelligence, qualitative becomes the most critical link. If the auto driving car with artificial intelligence system is recognized as "object" by law, because they can only drive automatically, and basically under the control of people, then the car accident in this kind of car should not be investigated for the responsibility of the car itself, because the car is only the carrier and only "thing". But because of the car accident, the problem of liability may be traced back to the user or operator. Other AI entities also have similar problems.

\section{The Significance and Necessity of Legal Regulation of Artificial Intelligence}

The original purpose of artificial intelligence research was to achieve human intelligence. Human intelligence is diverse, and it is able to make decision-making choices in the face of unspecified and uncertain problems. The frustrations of artificial intelligence research have made the current artificial intelligence research more inclined to the specialized field of intelligence. The general artificial intelligence is back to the original starting point of artificial intelligence, and re-pursuing the realization of human intelligence. The intelligence they want to achieve is also comprehensive, and cognition and learning become the most basic requirements of general artificial intelligence. Among them, the crisis and risk are deeply accompanied by the advancement of artificial intelligence technology. The development of the acceleration of technology has made artificial intelligence closer and closer to our lives, and it has more and more close relationships with us. In a legislative motion in May 2016, the EU mentioned that between 2010 and 2014, the sales of robots in the EU increased by an average of $17 \%$ per year, of which only sales in 2014 increased by $29 \%$ year-on-year, and annual patent applications for robotics have tripled in the past decade. 1 This also indicates that the field of artificial intelligence has flourished, and artificial intelligence has gradually occupied an important position in the production and life of our society. Behind the flourishing development is that we have to think about the status of these intelligent robots. Due to the risk of artificial intelligence is to some extent unbearable to our entire society. Therefore, it is necessary for us to properly manage the application of this technology. Moreover, artificial intelligence needs to go through social learning to some extent to gradually form its own relatively stable judgment. Any artificial intelligence researcher's designer creates artificial intelligence entities. These artificial intelligences only have the possibility of rational cognition and reasoning. They also need re-education of the social environment. If we human society treat them in an inhuman way, they may treat humans like this after learning how we behave. And this will be a disaster for humans and artificial intelligence, and perhaps a battle like humans and machines in the movie "Terminator" may break out. While human beings are standing at such crossroads, artificial intelligence can enhance our human intelligence, making human society develop abruptly, and human society may also explode. The key is how we regulate such a technology. The road to technological development may be unstoppable, but we have adopted laws to regulate such technologies as artificial intelligence, which is more conducive to the development of technology and more beneficial to human beings.

\section{ThE CHALLENGE OF ARTIFICIAL INTELLIGENCE TO THE CURRENT CHINA's PENALTY SYSTEM}

As we know, punishments in China are divided into principle punishments and supplementary punishments, the current Chinese penal system mainly includes control, detention, fixed-term imprisonment, life imprisonment, death penalty and other main sentences, as well as additional penalties, such as fine, deprivation of political rights, and confiscation of property. This kind of penalty setting is perfect and reasonable. But is the current Chinese penal system meaningful for Artificial intelligence?

Before we begin our discussion, we might as well assume that artificial intelligence has acquired legal qualification. On

http://www.europal.europa.eu/oeil/popups/ficheprocedure.do?lan $\mathrm{g}=$ en\&reference $=2015 / 2103$ (INL) 
this basis, we can discuss that whether different penalties can be effective for Artificial intelligence.

\section{A. China's Current Crime Law's Principle Punishments Applied to Artificial Intelligence}

The principle punishments include free penalty and death penalty. There can be no doubt that death penalty can be applied to Artificial intelligence-delete all data or remove program can be seen as the death penalty, because as far as the current scientific and technological level is concerned, we believe that all Artificial Intelligence rely on program to sustaining their survival. For special prevention, it can surely work. However, the capacity for suffering is not just another characteristic like the capacity for language or higher mathematics, considering the aim of general prevention, whether another Artificial intelligence can feel suffering is doubtful. But at least, the death penalty can work within a limit range. This means that for the current penalty system, the impact of artificial intelligence is more reflected in the level of free penalty. Therefore, the discussion on the principle punishment should focus on the level of current free penalty: in the case of artificial intelligence with subject consciousness, where is the space of application of free punishment, and can it effectively regulate artificial intelligence or not.

1) Current free penalty for artificial intelligence: Before discussing the limited free penalty, the first thing to be clear is that the regulation of artificial intelligence technology regulation is its procedure, not the carrier of its procedures. So we focus on how to limit the freedom of the program. From the perspective of China's existing free penalty, is it effective to put artificial intelligence into prison? To do this, we need to analyze what freedom means to artificial intelligence. The freedom of artificial intelligence should be the freedom of its survival and development. In simple terms, the survival and development of artificial intelligence depend on energy and data. Then its freedom should be based on the freedom of data analysis and processing in the context of energy supply. From this point of view, is China's existing free penalty effective? It is objectively said that it has a certain degree of applicability, and an artificial intelligence product is directly thrown into prisons and other institutions, forcing it to comply with certain regulations can't be said to be worthless, but this kind of restriction is not considering the characteristics of artificial intelligence. The freedom of artificial intelligence is obviously different from the personal freedom that the current free penalty relies on. Therefore, from a big conceptual point of view, free punishment may have an effect on artificial intelligence, but the effect needs to be accurately grasping the artificial intelligence characteristics and updating the penalty execution method, and constructing a new form of free punishment.

2) The envisaged free penalty for artificial intelligence: As mentioned above, artificial intelligence relies on energy and data processing to build the freedom of data analysis and processing. From the perspective of energy and data, perhaps we can explore a more effective way to rule Artificial Intelligence.

Energy. We can use electrical energy as the main discussion object, and the data generally includes local data and external data connection. The former relies on the storage space of the artificial intelligence carrier, and the latter is mainly realized through the network connection. From this point of view, for artificial intelligence, the most suitable for free penalty construction should be power outages, limit reading and writing, and disconnection. In terms of power outage, it is nothing more than a further extension of the shutdown operation of intelligent electronic devices at this stage. Analogy to humans is more like sleeping. Perhaps the mandatory sleep can have some degree of freedom, but once the power is cut off, the artificial intelligence's perception will be immediately lost, and in the case of loss of perception, it can't be painful. How can the purpose of punishment be realized? In terms of restricting reading and writing, it can be divided into the limitation of authority and the limitation of ability. Comparing with human life, the former can be analogized to the limits and deprivations of certain qualifications, while the latter can be analogized to reduce human capabilities through some means. For the former, the actual validity of the qualification penalty has shown to be effective, but for the latter, in the case of reduced ability, things that Artificial Intelligence can understand before becoming incomprehensible after, and whether it is certain that suffering can be felt is uncertain. In the case of a broken network, can we only disconnect the network link to limit its freedom? Considering the existence of local storage, this limitation is limited. Accordingly, for the free restriction of artificial intelligence, it is more appropriate to disconnect the network link and limit the interaction between reading and writing.

At the same time, starting from the working mode of artificial intelligence, we can see that the "survival" of artificial intelligence depends on the commands of the program written in its program code. Since we assume it as an independent entity the survival and development of this subject is based on the command statement. In this perspective, artificial intelligence is modified by high-level command statements, limiting its read and write behavior progresses. We can think of it as a restriction on its freedom.

\section{B. China's Current Crime Law's Supplementary Punishments Applied to Artificial Intelligence}

China's additional punishment consists of deprivation of political rights, fines, and confiscation of property. It is not difficult to find that whether it is political rights or property rights, the premise of artificial intelligence is that artificial intelligence has the legal qualification of the subject. Starting from the criminal law theory of civil law, it is obviously more lenient than the criminal law to identify the civil law of responsibility. But if so, artificial intelligence organisms will have basic rights such as the right to life and property rights. Looking back at history, even the cloning technology that only copies the living human body has been subjected to fierce criticism in the field of ethics and sociology. So far, 
clones have not been able to produce, and their main qualifications. It's hard to imagine that at the level of artificial intelligence, we can think that a collection of programs consisting of code and almost no life representation can have the potential to become "people". Unless the artificial intelligence can be called a "person", the supplementary punishments under the current penal system has no room for application.

Combined with the discussion of the current criminal law on the main and additional penalties, we can further conclude that if a subject cannot be recognized as a civil subject within the scope of a broader civil law, it will be hard to regulate AI through supplementary punishments. Thus, before solving the grand proposition that artificial intelligence has civil legal qualifications, the current supplementary punishments system is difficult to effectively regulate artificial intelligence.

\section{CONCLUSION}

To sum up, based on artificial intelligence technology, it is feasible in theory to regulate artificial intelligence by law. However, it is impossible to regulate artificial intelligence effectively, whether it is the current main punishment or the existing supplementary punishment system in China. We should also innovate the independent and brand-new punishment that belongs to the human intelligence. This is an inevitable choice for mankind to effectively control AI and bring it into the path conducive to mankind. But it is still worth thinking about whether the punishment of artificial intelligence can make artificial intelligence feel pain, especially whether it can really play a special precaution against other artificial intelligence. It is believed that with the progress of artificial intelligence technology, people can master the generation and mechanism of the "emotion" of artificial intelligence, which can be solved sooner or later. Penalty regulation of artificial intelligence is not only a question that China should think about, but also a global problem. In the future, no matter whether the current concept is realized or not, we cannot deny the significance of forward-looking thinking. It's important to achieve the balance between artificial intelligence and human.

\section{REFERENCES}

[1] The Criminal Liability of Artificial Intelligence Entities From Science Fiction to Legal Social Control. Gabriel Hallevy. Akron Intell.Prop.J . 2010

[2] Toward the Human-Robot Co-Existence Society: On Safety Intelligence for Next Generation Robots. Yueh-Hsuan Weng,ChienHsun Chen,Chuen-Tsai Sun. International Journal of Social Robotics 2009

[3] Intelligence and Social Inequality:Why the Biological Link. Linda Gottfredson. . 2010

[4] Super Intellgence:Paths Dangers Strategies. Nick Bostrom. . 2014

[5] The robot that takes your job should pay taxes says Bill Gates. Kevin J Delaney. Quartz . 2017

[6] "Data Protection for the Digital Age:Comprehensive Effects of the Evolving Law of Accountability", Nina Gumzej. Juridical Tribune. 2012
[7] "An Ethical Obligation to Use Artificial Intelligence?An Examination of the Use of Artificial Intelligence in Law and the Model Rules of Professional Responsibility". Andrew Arruda. American Journal of Trial Advocacy . 2016

[8] AI:Legal Research and Law Librarians. Sherry Xinchen,Mary Ann Neary. AALL Spectrum . 2016-2017

[9] Artificial Intelligence Role in Cybersecurity Infrastructures. Cristian Vlad Oancea. Int' '1 J.Info.Sec.\&Cybercrime . 2015

[10] Regulating Artificial Intelligence Systems:Risks, Challenges, Compe 